\title{
Association between adherence to dietary recommendations and high-sensitivity C-reactive protein level in type 1 diabetes
}

\author{
Ahola, Aila J. \\ 2017-04
}

Ahola , A J , Saraheimo , M , Freese , R, Forsblom , C, Mäkimattila , S , Groop , P-H \& FinnDiane Study Grp 2017 , ' Association between adherence to dietary recommendations and high-sensitivity C-reactive protein level in type 1 diabetes ' , Diabetes Research and Clinical Practice , vol. 126 , pp. 122-128 . https://doi.org/10.1016/j.diabres.2017.02.012

http://hdl.handle.net/10138/237093

https://doi.org/10.1016/j.diabres.2017.02.012

unspecified

publishedVersion

Downloaded from Helda, University of Helsinki institutional repository.

This is an electronic reprint of the original article.

This reprint may differ from the original in pagination and typographic detail.

Please cite the original version. 


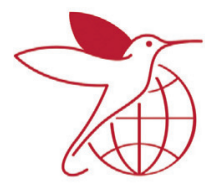

International

Diabetes

Federation

\title{
Association between adherence to dietary recommendations and high-sensitivity C-reactive protein level in type 1 diabetes
}

\author{
Aila J. Ahola ${ }^{a, b, c}$, Markku Saraheimo ${ }^{a, b, c}$, Riitta Freese ${ }^{d}$, Carol Forsblom ${ }^{a, b, c}$, \\ Sari Mäkimattila ${ }^{b}$, Per-Henrik Groop ${ }^{a, b, c, e, *}$, on behalf of the FinnDiane Study Group \\ ${ }^{a}$ Folkhälsan Institute of Genetics, Folkhälsan Research Center, Helsinki, Finland \\ ${ }^{\mathrm{b}}$ Abdominal Center Nephrology, University of Helsinki and Helsinki University Central Hospital, Helsinki, Finland \\ ${ }^{\mathrm{c}}$ Research Program Unit, Diabetes and Obesity, University of Helsinki, Finland \\ ${ }^{\mathrm{d}}$ Division of Nutrition, Department of Food and Environmental Sciences, University of Helsinki, Finland \\ e Baker IDI Heart and Diabetes Institute, Melbourne, Victoria, Australia
}

\section{A R T I C L E I N F O}

Article history:

Received 29 September 2016

Accepted 7 February 2017

Available online 15 February 2017

Keywords:

Adherence to dietary

recommendations

hs-CRP

Low-grade inflammation

Type 1 diabetes

\begin{abstract}
A B S T R A C T
Aims: Inflammation plays an important role in the pathogenesis of cardiovascular diseases. Diet, as a modifiable risk factor, may in turn impact systemic inflammation. We therefore assessed whether adherence to the dietary recommendations is associated with high-sensitivity C-reactive protein (hs-CRP) concentrations in type 1 diabetes.

Methods: Cross-sectional data from 677 FinnDiane study participants (48\% men, mean \pm standard deviation age $46 \pm 13$ years) were included. Dietary intake was assessed with a self-administered questionnaire. A diet score, with higher values denoting better adherence to the recommendations, was calculated. Serum hs-CRP concentration was measured, and individuals with hs-CRP $<1.0 \mathrm{mg} / \mathrm{l}$, and hs-CRP $>3.0$ but $\leq 10.0 \mathrm{mg} / \mathrm{l}$ were compared.

Results: Men and women with high hs-CRP had higher BMI, waist circumference, and triglyceride concentration, but lower HDL-cholesterol concentration. Adjusted for BMI, mean diet score was higher in the low hs-CRP group, both in men $(10.8 \pm 3.6$ vs. $9.9 \pm 3.8$, $p=0.023)$ and women $(12.7 \pm 3.4$ vs. $11.6 \pm 3.5, p=0.021)$. After further adjustments with potential confounding factors, the difference remained significant only in men.

Conclusions: A diet that more closely adheres to the dietary recommendations is associated with lower hs-CRP in men. A prudent diet may help reduce systemic inflammation in type 1 diabetes.
\end{abstract}

(c) 2017 Elsevier B.V. All rights reserved.

\section{Introduction}

Individuals with diabetes have an increased risk of developing various micro- and macrovascular complications [1]. Impor- tantly, the long-term complications account for the majority of the increased morbidity and mortality related to diabetes [2]. To reduce human suffering and the burden the treatment of these complications pose on the health care system, any

\footnotetext{
* Corresponding author at: Folkhälsan Research Center, Biomedicum Helsinki C318b, PO Box 63, 00014 University of Helsinki, Finland. Fax: +358919125452.

E-mail address: per-henrik.groop@helsinki.fi (P.-H. Groop). 
actions to prevent the development of diabetic complications should be considered.

Inflammatory processes play an important role in the pathogenesis of cardiovascular diseases. Indeed, elevation of inflammatory biomarkers, such as C-reactive protein (CRP), in individuals with acute myocardial infarction is well described [3,4]. Moreover, in a meta-analysis, every 1standard deviation increase in the CRP concentration was associated with a $37 \%$ and $27 \%$ increase in the risk of coronary heart disease and ischaemic stroke, respectively [5]. It is of note that inflammatory mechanisms can produce atherosclerotic changes even in the absence of traditional risk factors [6]. Moreover, we have previously shown in the FinnDiane Study, that low-grade inflammatory markers are associated with diabetic nephropathy in type 1 diabetes [7].

Diet is a modifiable risk factor that has been related to systemic inflammation. In the NHANES study, conducted in the general population, the highest scores in the Healthy Eating Index, as opposed to the lowest scores, were independently associated with lower CRP concentrations in men but not in women [8]. In a randomised controlled trial, in individuals at high risk of cardiovascular disease, consumption of a Mediterranean diet led to beneficial effects on blood pressure, lipid profile, measures of carotid atherosclerosis, and inflammation [9]. Finally, during a 7-year follow-up, consumption of fresh fruits had favourable, whereas consumption of total and high-fat dairy products had unfavourable impact on lowgrade inflammation amongst individuals with type 2 diabetes and/or cardiovascular disease [10].

Not many studies have been conducted in patients with type 1 diabetes. Importantly, rather than exploring the overall quality of the diet, these studies have focused on a few selected nutrients. Two cross-sectional studies evaluated the associations of dietary fibre with systemic inflammation and showed mixed results [11,12]. In a large sample of youth with type 1 diabetes, dietary fibre intake was not associated with systemic inflammation [11], while a high fibre diet was associated with lower hs-CRP in a small sample of adult participants [12]. In the EURODIAB study, lower consumption of fibre and polyunsaturated fats, but higher consumption of cholesterol were, over an 8 year study period, associated with higher levels of low-grade inflammation [13]. Finally, in a group of patients with type 1 diabetes, Strychar et al. compared the effects of two isocaloric diets on a number of variables including inflammatory markers over 6 months [14]. One diet was higher in carbohydrate and lower in fat content, and the other was lower in carbohydrates and higher in monounsaturated fats. While there was no difference in inflammation, between the two groups, the diet lower in carbohydrates and higher in monounsaturated fats resulted in lower triglyceride and lower postprandial glucose concentrations.

This study was conducted to further explore the potential role of diet on inflammation in type 1 diabetes. We studied the association between adherence to the dietary recommendations and low-grade inflammation. We hypothesised that closer adherence to the dietary recommendations would be associated with a lower level of low-grade inflammation, measured as hs-CRP concentration.

\section{Methods}

\subsection{Study subjects}

Study subjects were participants in the Finnish Diabetic Nephropathy (FinnDiane) Study. The FinnDiane Study was established with the aim to reveal factors associated with the emergence of diabetic complications, especially diabetic nephropathy, in patients with type 1 diabetes. The study was launched in 1997 with ongoing recruitment. The diet sub-study was inaugurated in 2007. Included in the current cross-sectional analyses were all participants $(n=677)$ in the FinnDiane Study who had filled in the diet questionnaire within two years from their study visit and had hs-CRP concentration either below $1.0 \mathrm{mg} / \mathrm{l}$ (low hs-CRP level) or between $>3.0$ and $\leq 10.0 \mathrm{mg} / \mathrm{l}$ (high hs-CRP level). These cut off values were selected as they are considered to represent those at low and high risk of future cardiovascular events, respectively [15]. However, individuals with hs-CRP values $>10 \mathrm{mg} / \mathrm{l}$ were excluded as they were likely to have had an infection. Participant selection is shown in Fig. 1. The study protocol was approved by the Ethics Committee of the Helsinki University Central Hospital. Study subjects provided written informed consent prior to participation.

\subsection{Dietary intake}

Participants' habitual dietary intake was assessed with a selfadministered diet questionnaire [16]. In short, the questionnaire was designed to capture the consumption pattern of

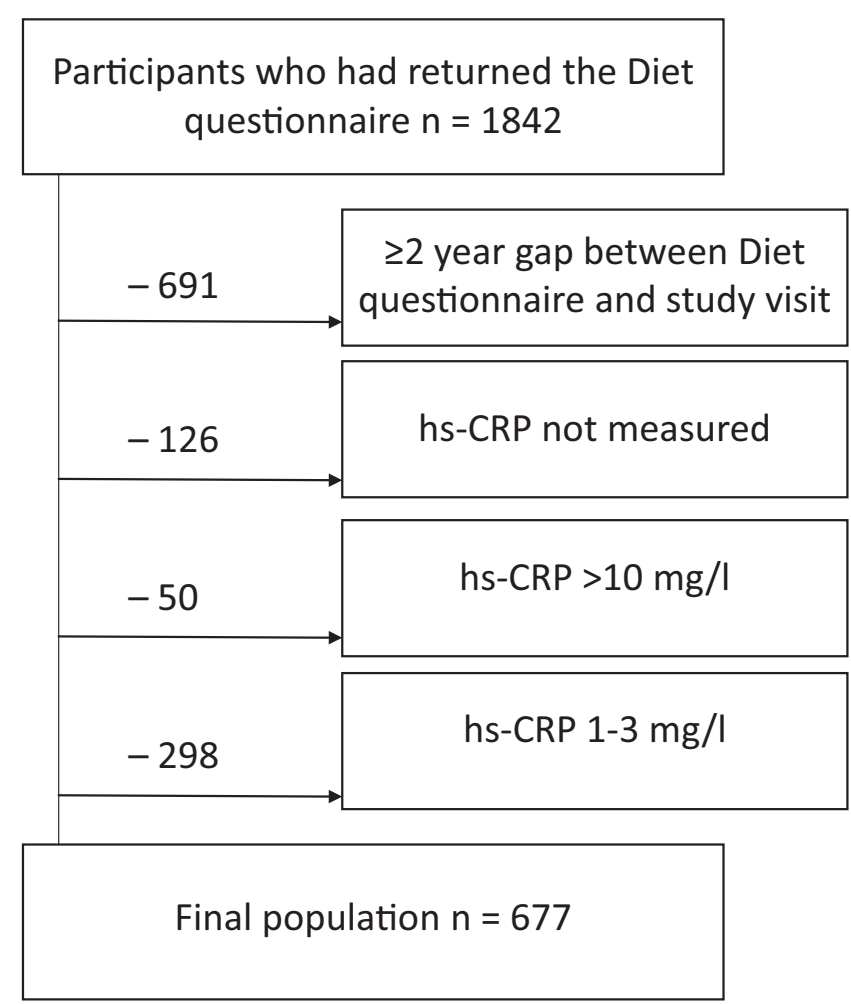

Fig. 1 - Flow chart of the study population. 
the most typical food items consumed in Finland. Amongst others, included were questions about coffee, milk, bread, spread, and cooking fat consumption. Included was also a section with a food frequency questionnaire where the consumption frequencies of various food items, such as fish, meat, poultry, vegetables, fruits and berries, and soft drinks, were queried. The validity of the diet questionnaire has been investigated, and it was observed to show reasonable relative validity against a 6-day food record (unpublished data). Entries in the diet questionnaires were compared against the dietary recommendations for patients with diabetes [17] and a diet score was calculated [18]. The principals for calculating the score are presented in Supplemental Table 1. Briefly, the consumption patterns of 11 dietary components were included in the calculation of the score: fish, fresh vegetables, cooked vegetables, fruits and berries, soft drinks, sweet pastry, candy, low-fat liquid milk products, vegetable based spreads, vegetable oil based cooking fats, and salt. Those more closely adhering to the individual dietary recommendation, related to these above-mentioned food items, scored 2 points, while those whose adherence was the worst, scored 0 points. The potential diet scores range between 0 and 22, the highest scores represent individuals most closely complying with the dietary recommendations.

Upon returning the diet questionnaire, participants were sent an exercise and diet record, as previously described [16]. In this record, all foods and drinks consumed over the allocated three days were reported. Moreover, in the same form, participants reported their leisure-time physical activities, measured blood glucose values and insulin administration. The record-keeping was repeated in 10 weeks. Up to 2 reminders were sent to the non-responders. Daily energy intake, reported in the record, was calculated using Diet 3.2 software (version 1.4.6.2, AIVO, Turku, Finland) and, from August 2014 onwards, AivoDiet software (version 2.0.2.3, AIVO, Turku, Finland). Both of these programs are based on the Finnish National Food Composition Database, Fineli.

\subsection{Physical activity}

The level of leisure time physical activity (LTPA) was assessed with a questionnaire, as previously described [19]. In this form, mean frequency, single session duration, and intensity of 21 most common forms of physical activity were reported for the preceding 12 months. The total amount of LTPA per week was calculated by multiplying the duration of the activity by the activity- and intensity-specific metabolic equivalent (MET). The weekly MET hour (MET h) value was treated as a continuous variable in the analyses.

\subsection{Clinical measurements}

Height and weight were measured in light clothing, and body mass index (BMI) was calculated $\left(\mathrm{kg} / \mathrm{m}^{2}\right)$. Following at least a 10-min rest, blood pressure was measured in the sitting position. The measurement was repeated after $2 \mathrm{~min}$. The mean of these measurements was used in the analyses. Serum hsCRP concentration was measured by immunoassay (Modular analyzer, Roche). $\mathrm{HbA}_{1 \mathrm{c}}$ was determined locally using standardized assays. Serum lipid and lipoprotein concentrations were measured as previously described [20]. Renal status was assessed based on the urinary albumin excretion rate (AER) in at least two out of three timed 24-h or overnight urine collections. Patients were classified as having normal albumin excretion rate (AER $<20 \mu \mathrm{g} / \mathrm{min}$ or $<30 \mathrm{mg} / 24 \mathrm{~h}$ ), microalbuminuria (AER $\geq 20$ and $<200 \mu \mathrm{g} / \mathrm{min}$ or $\geq 30$ and $<300 \mathrm{mg} / 24 \mathrm{~h}$ ), macroalbuminuria (AER $\geq 200 \mu \mathrm{g} / \mathrm{min}$ or $\geq 300 \mathrm{mg} / 24 \mathrm{~h}$ ), or end-stage renal disease (ESRD) (undergoing dialysis or having had a kidney transplant). Diabetic nephropathy was defined as macroalbuminuria or ESRD. Smoking was self-reported.

\subsection{Statistical analyses}

Descriptive statistics are reported as median (interquartile range) for continuous non-normally distributed data, mean \pm standard deviation for continuous normally distributed data, and percentages for categorical data. The respective between-group comparisons were conducted with MannWhitney U-test, independent samples t-test, and Chisquared test (two-sided tests). The Spearman correlation coefficient was calculated to study the correlations between the hs-CRP concentration and the diet score. Analysis of covariance was used to study the association between the hs-CRP status and the diet score. Significant basic characteristics (from Table 1) were considered as cofactors for the multivariable analyses when studying the independent associations between the diet score and the hs-CRP status. Prior to inclusion, their inter-correlation was first studied with Pearson correlation coefficient, and following the initial variable selection, a linear regression analysis was conducted (collinearity diagnostics) to ensure that no multicollinearity was present. All data were analysed using IBM SPSS Statistics for Windows, Version 22.0 (IBM Corp, Armonk, NY, USA). A $p$ value $<0.05$ was considered statistically significant.

\section{Results}

Data from a total of 677 individuals (48\% men, mean \pm standard deviation age $46 \pm 13$ years) were available for the analyses. The median (IQR) hs-CRP concentration was higher in women than in men $[0.79(0.42-4.07)$ vs. $0.60(0.36-0.95)$, respectively, $p<0.001$ ]. A total of $47 \%$ and $65 \%$ of the individuals in the low hs-CRP and the high hs-CRP group, respectively, were women $(p<0.001)$. The median diet score in men and women differed significantly [11 (8-13) vs. 12 (10$15)$, respectively, $p<0.001$ ]. Finally, the association between hs-CRP and diet score differed significantly between the sexes ( $p$-value for the interaction $<0.001$ ). Due to these differences, data from men and women were analysed separately.

In men and women, the high hs-CRP group had higher mean BMI, waist circumference, and triglyceride concentration, but lower HDL-cholesterol concentration and physical activity level (Table 1). Men in the high hs-CRP group reported lower energy intake, while women in the high hs-CRP group were younger, had worse glycaemic control, and higher diastolic blood pressure. In women, the hs-CRP concentration correlated negatively with the diet score $(r=-0.146, p=0.006)$, while in men, no such correlation was observed $(r=-0.067$, $p=0.229$ ). 
Table 1 - Basic characteristics in men and women divided by the hs-CRP status.

\begin{tabular}{|c|c|c|c|c|c|c|}
\hline & \multicolumn{3}{|l|}{ Men } & \multicolumn{3}{|l|}{ Women } \\
\hline & $\begin{array}{l}\text { Low hs-CRP } \\
N=250\end{array}$ & $\begin{array}{l}\text { High hs-CRP } \\
N=73\end{array}$ & $p$ & $\begin{array}{l}\text { Low hs-CRP } \\
N=219\end{array}$ & $\begin{array}{l}\text { High hs-CRP } \\
N=135\end{array}$ & $p$ \\
\hline hs-CRP, mg/l & $0.49(0.30-0.71)$ & $4.56(3.68-5.95)$ & $<0.001$ & $0.51(0.32-0.73)$ & $4.62(3.50-6.33)$ & $<0.001$ \\
\hline Diet score & $11(8-13)$ & $10(7-12)$ & 0.062 & $13(10-15)$ & $12(9-14)$ & 0.003 \\
\hline Energy intake, kcal & $2149 \pm 569$ & $1904 \pm 586$ & 0.006 & $1759 \pm 444$ & $1721 \pm 387$ & 0.467 \\
\hline Age, years & $45.4 \pm 13.0$ & $47.6 \pm 12.1$ & 0.198 & $47.5 \pm 12.7$ & $42.1 \pm 13.2$ & $<0.001$ \\
\hline Duration, years & $26.9 \pm 14.0$ & $29.9 \pm 13.4$ & 0.100 & $29.6 \pm 13.1$ & $27.9 \pm 13.6$ & 0.238 \\
\hline Current smoking, \% & 14.8 & 13.0 & 0.846 & 11.9 & 17.6 & 0.153 \\
\hline Weekly MET h & $13(0-30)$ & $7(0-20)$ & 0.040 & $16(0-31)$ & $7(0-16)$ & $<0.001$ \\
\hline $\mathrm{HbA}_{1 \mathrm{c}}, \mathrm{mmol} / \mathrm{mol}$ & $63(55-72)$ & $65(57-73)$ & 0.240 & $62(56-71)$ & $71(61-80)$ & $<0.001$ \\
\hline $\mathrm{HbA}_{1 \mathrm{c}}, \%$ & $7.9(7.2-8.7)$ & $8.1(7.4-8.8)$ & 0.240 & $7.8(7.3-8.6)$ & $8.6(7.7-9.5)$ & $<0.001$ \\
\hline BMI, $\mathrm{kg} / \mathrm{m}^{2}$ & $24.8 \pm 3.0$ & $28.2 \pm 4.6$ & $<0.001$ & $24.0 \pm 2.9$ & $28.0 \pm 4.8$ & $<0.001$ \\
\hline Waist circumference, cm & $88(83-95)$ & $103(94-111)$ & $<0.001$ & $78(73-84)$ & $89(82-97)$ & $<0.001$ \\
\hline $\mathrm{SBP}, \mathrm{mmHg}$ & $138(128-152)$ & $141(130-154)$ & 0.718 & $132(121-144)$ & $134(120-144)$ & 0.663 \\
\hline DBP, mmHg & $77 \pm 10$ & $79 \pm 11$ & 0.121 & $74 \pm 8$ & $78 \pm 10$ & $<0.001$ \\
\hline Hypertension, \% & 84.3 & 88.7 & 0.448 & 67.4 & 80.3 & 0.010 \\
\hline Triglycerides, mmol/l & $0.90(0.71-1.27)$ & $1.22(0.90-1.85)$ & $<0.001$ & $0.72(0.57-0.92)$ & $1.10(0.86-1.56)$ & $<0.001$ \\
\hline Total cholesterol, $\mathrm{mmol} / \mathrm{l}$ & $4.31 \pm 0.87$ & $4.46 \pm 1.00$ & 0.254 & $4.59 \pm 0.84$ & $4.64 \pm 0.98$ & 0.668 \\
\hline HDL cholesterol, $\mathrm{mmol} / \mathrm{l}$ & $1.52 \pm 0.43$ & $1.37 \pm 0.39$ & 0.017 & $1.85 \pm 0.46$ & $1.64 \pm 0.45$ & $<0.001$ \\
\hline Lipid-lowering medication, \% & 34 & 56 & 0.001 & 27 & 36 & 0.091 \\
\hline Diabetic nephropathy, \% & 22 & 34 & 0.051 & 12 & 17 & 0.314 \\
\hline Any hard CV event, \% & 14 & 22 & 0.096 & 8 & 13 & 0.199 \\
\hline \multicolumn{7}{|c|}{$\begin{array}{l}\text { Data are presented as median (interquartile range) for continuous non-normally distributed variables, mean } \pm \text { SD for continuous normally } \\
\text { distributed variables, and frequency (\%) for categorical variables. Low hs-CRP, high-sensitive C-reactive protein concentration }<1.0 \mathrm{mg} / \mathrm{l} \text {; High } \\
\text { hs-CRP, high-sensitive C-reactive protein concentration between }>3.0 \text { and } \leq 10.0 \mathrm{mg} / \mathrm{l} \text {; MET } \mathrm{h} \text {, metabolic equivalent of task hours; BMI, body } \\
\text { mass index; SBP, systolic blood pressure; DBP, diastolic blood pressure; Hypertension, SBP } \geq 130 \mathrm{mmHg} \text { or DBP } \geq 85 \mathrm{mmHg} \text { or antihypertensive } \\
\text { medication; Any hard CV event, acute myocardial infarction, coronary bypass, stroke, amputation, or peripheral vascular disease. }\end{array}$} \\
\hline
\end{tabular}

Table 2 - Diet scores in men and women divided by hs-CRP status.

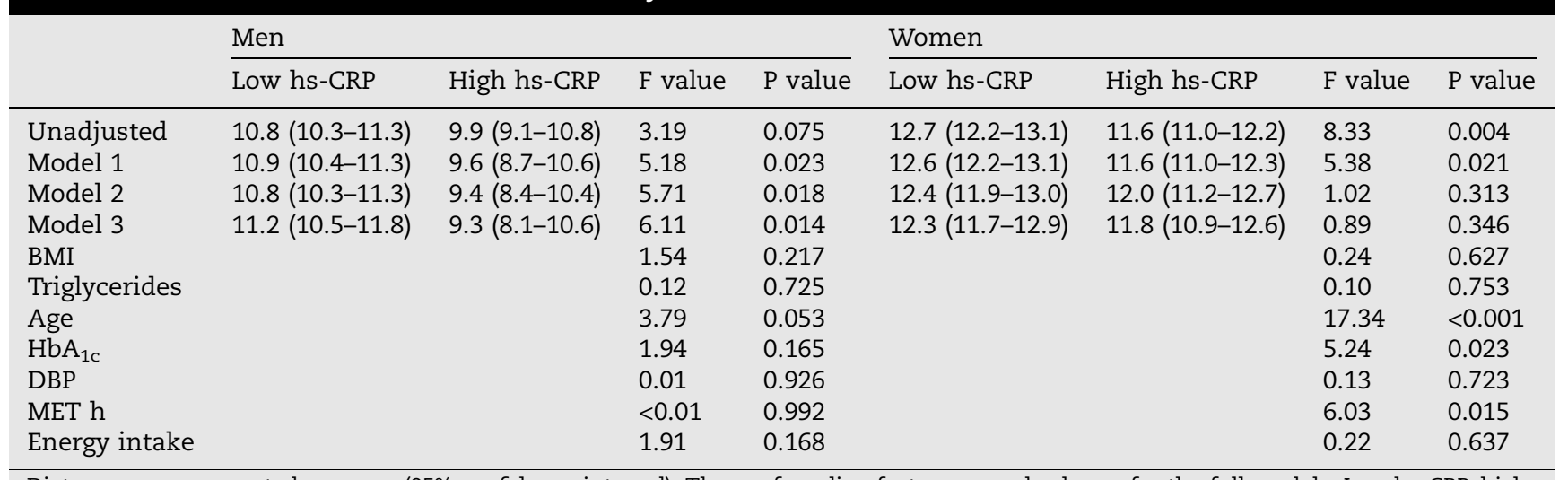

Diet scores are presented as mean (95\% confidence interval). The confounding factors are only shown for the full models. Low hs-CRP, highsensitive C-reactive protein concentration $<1.0 \mathrm{mg} / \mathrm{l}$; High hs-CRP, high-sensitive C-reactive protein concentration between $>3.0$ and $\leq 10.0 \mathrm{mg} / \mathrm{l}$; BMI, body mass index; DBP, diastolic blood pressure; MET h, metabolic equivalent of task hours. Analysis of covariance.

Model 1 adjusted for BMI.

Model 2 additionally adjusted for triglyceride concentration, age, $\mathrm{HbA}_{1 \mathrm{c}}$, DBP.

Model 3 additionally adjusted for physical activity and energy intake.

Adjusted for BMI, the diet score was significantly higher in the low hs-CRP groups, both in men and women (Table 2), suggesting that individuals with lower hs-CRP levels more closely adhered to the dietary recommendations. After fur- ther adjustments for age, triglyceride concentration, $\mathrm{HbA}_{1 \mathrm{c}}$, diastolic blood pressure, physical activity and energy intake, the difference in the diet scores, between the two hs-CRP groups remained significant only in men. 


\section{Discussion}

In the current analyses amongst a population of well-defined individuals with type 1 diabetes, men, but not women, with lower hs-CRP concentrations were observed to more closely adhere to the dietary guidelines. The reason for the observed sex difference in the association between diet score and inflammation is not known. However women, on average, had higher diet scores than men. Of the individual components of the diet score, compared to men, women reported higher median intakes of fresh vegetables, cooked vegetables, and fruits and berries. Potentially higher intake of antioxidants, amongst women, may mask the association between diet score and hs-CRP that was observed in men. Interestingly, the observed gender difference remained also in a sub-analysis, where all individuals with hs-CRP concentration $\leq 10 \mathrm{mg} / \mathrm{l}(n=975)$ were included (data not shown).

Our results are in concordance with the results from the National Health and Nutrition Examination Survey (NHANES), where the poorest diet quality was associated with elevated CRP concentrations in men but not in women [8]. Similarly to our study, a diet score based on the level of adherence to the dietary guidelines, to describe the overall quality of the diet, was also used in the NHANES. Common to both scores, fruits, vegetables, milk products, vegetable oil based fats, sugary items, and salt were taken into account. Some differences in the included food items existed, however. Our score, for example, also included the consumption of fish, while we did not include the use of grains. Despite their differences, both scores were positively associated with a lower level of low-grade inflammation.

A prudent diet, characterized by higher intake of fruits, vegetables, legumes, fish, poultry, and whole grains was, in the Nurses' Health Study, inversely associated with the CRP concentrations [21]. Also a Mediterranean-style diet pattern, characterized by ample intake of whole grains, fruits, vegetables, nuts, and olive oil, has been associated with lower levels of low-grade inflammation [9].

Similarly to the current study, association between diet and low-grade inflammation in patients with type 1 diabetes was also studied in the EURODIAB study [13]. In their study, a three-day food record was completed at baseline and after an 8-year follow-up period to assess the dietary intake of the subjects across 16 European countries. According to the results, a number of dietary factors, generally regarded as "healthy", were associated with a lower level of systemic inflammation. Indeed, those consuming more total fibre, more soluble fibre, more polyunsaturated fats, but less cholesterol over the 8-year study period, had lower levels of low-grade inflammation.

There may be several components in a prudent diet that favourably affect the inflammatory status. As low-grade inflammation is related to the markers of oxidative stress [22], some of the components of a healthy diet could function as antioxidants and thereby reduce inflammation. Related to this, Wood et al. observed that a high serum carotenoid concentration, a biomarker for fruit and vegetable consumption, was associated with lower systemic inflammation [23]. Vegetable oils, whole grains, and nuts are rich sources of vitamin
$E$, the intake of which increases the serum $\alpha$-tocopherol concentration. Increasing quintiles of serum $\alpha$-tocopherol concentration has been associated with decreasing levels of inflammation [23]. Consumption of fish oil, rich in omega-3 fatty acids, has also been shown to decrease the serum CRP concentrations [24]. A prudent diet is typically low in trans fatty acids, which have been shown to have proinflammatory effects and promote endothelial dysfunction [25]. Finally, dietary fibre may mediate inflammatory processes via intestinal production of short-chain fatty acids. Butyrate, for example, has been shown to inhibit the TNF $\alpha$ induced activation of nuclear factor-kappa B, a key transcription factor in the regulation of genes related to inflammatory responses [26].

In the current study, larger waist circumference, and higher BMI were observed in the high hs-CRP groups, both in men and women. Importantly, obesity and overweight are associated with insulin resistance, which has previously been shown to be associated with increased plasma hs-CRP concentrations [27]. Moreover, increased adiposity is also associated with less advantageous inflammatory profile $[23,28]$. Due to strong correlation between BMI and waist circumference, we chose to include only BMI in the multivariable model. We did, however, run the analysis with waist circumference, and the results remained unchanged. In the final models, neither BMI nor waist circumference was independently associated with the inflammation status.

Beyond diet, other life style factors may also mediate inflammation. In a recent meta-analysis of 43 trials Hammonds et al. for example, demonstrated the inflammationreducing effects of exercise interventions [29].The mechanisms by which physical activity reduces inflammation is not clear, however. Proposed mechanisms include decreased body weight and fatness, and improved insulin sensitivity [30]. Based on the inflammation-reducing effects of physical activity and healthy diet, it may be speculated that individuals in high risk of cardiovascular disease could benefit from intensified health-care management programs, as the one reported by Ciccone et al. [31].

Hyperglycaemia is also known to induce inflammation [32]. In concordance with the observations made by Jarvandi et al. [8], worse glycaemic control in women was, in the current analyses, independently associated with being in the high hs-CRP group. Why this was not seen in men is not known, but could have partly been due to a low number of men in the high hs-CRP group.

Interestingly younger age, in women, was independently associated with being in the high hs-CRP group. The reason for this is not known. If something, we would have expected to see older age to be associated with higher level of inflammation. Indeed, aging is associated with a number of alterations in the immune system that are accompanied by the increased production of inflammatory cytokines [33]. While the age difference between the two groups, in the current study, was statistically significant, the difference may not be clinically significant to show any age-related changes in the immune system.

Our study has several limitations that need to be addressed. First, the data are cross-sectional, which forbids 
us to draw any conclusions about the causal relationships between the variables. While it is biologically plausible that adhering to a healthy diet may reduce low-grade inflammation, it is also possible that conditions associated with lowgrade inflammation have brought about changes in dietary practices. However, low-grade inflammation per se is not typically associated with marked symptoms that could be related to an obvious need to make alterations to one's diet. Similarly, it cannot be excluded that some unmeasured factors, unrelated to the diet, may be responsible for the observed hsCRP concentrations. Second, individuals volunteering in studies may not be representative of the general population. Indeed, those participating in studies may be more health conscious, with healthier dietary habits and a tendency to more actively seek help for various health conditions, including those related to inflammation. If such a selection bias has taken place in our study, this could have diluted the current observations. Third, as is often the case in epidemiological studies, dietary data were collected using a self-reported method. These methods are prone to bias, as participants may have a tendency to over-report food stuffs that are considered healthy, and underreport those considered unhealthy. While it could be speculated that such bias may have taken place in both hs-CRP groups alike, it has to be acknowledged that there were a number of other differences between the two groups that could contribute to an uneven reporting of dietary intake. For example, the distribution of obese individuals was not even between the groups. Related to this, the problem of underreporting of energy intake amongst obese individuals is a well acknowledged phenomenon [34]. However, if such bias has taken place it has, again, most likely diluted our results. Fourth, calculation of the diet score was based on the current dietary recommendations for individuals with diabetes. Although our results showed that the more closely the participants followed the dietary recommendations the lower the low-grade inflammation, this does not exclude the possibility that another diet pattern could exist that would be associated with even lower level of low-grade inflammation. Thus, our results do not suggest that a diet that adheres to the current recommendations is the single best dietary approach to tackle low-grade inflammation. To study the effect of a particular diet on inflammation, a double-blinded randomized interventional trial would be required.

In conclusion, a diet that more closely adheres to the current dietary recommendations for patients with diabetes was, in men, associated with a lower level of low-grade inflammation. Thus, our results suggest that consumption of fish, vegetables, fruits and berries, low-fat liquid milk products, and vegetable oil-based fats for spreads and for cooking, and limited intake of sweet pastry, candy, soft drinks, and salt may help to reduce systemic inflammation in type 1 diabetes. Given the importance of systemic low-grade inflammation on the cardiovascular health, our results also suggest that dietary approaches may be used as a means to reduce the risks of long term complications. In order to confirm the causal effect of diet on low-grade inflammation, however, randomised controlled trials are needed.

\section{Conflict of interest}

Professor Per-Henrik Groop has received research grants from Eli Lilly and Roche, is an advisory board member for AbbVie, Astra Zeneca, Boehringer-Ingelheim, Cebix, Eli Lilly, Janssen, MSD, Medscape, Novartis. He has received lecture fees from Astra Zeneca, Boehringer-Ingelheim, Eli Lilly, Genzyme, MSD, Novartis, Novo Nordisk, and Sanofi. All other authors declare that they have no conflict of interest.

\section{Ethics approval}

All procedures performed in studies involving human participants were in accordance with the ethical standards of the institutional and/or national research committee and with the 1964 Helsinki declaration and its later amendments or comparable ethical standards.

\section{Acknowledgements}

This study was supported by grants from Academy of Finland, Novo Nordisk Foundation, Signe and Ane Gyllenberg Foundation, Folkhälsan Research Foundation, Wilhelm and Else Stockmann Foundation, Liv och Hälsa Foundation, and the Helsinki University Central Hospital Research Funds (EVO). Funding agencies played no role in defining the study design: in the data collection, analysis and interpretation of data; in the writing of the manuscripts; or in the decision to submit the manuscript for publication. The skilled technical assistance of Anna Sandelin, Jaana Tuomikangas, and Satu Kinnunen is gratefully acknowledged. Finally, the authors acknowledge all the physicians and nurses at each centre participating in the collection of patients (online appendix).

\section{Appendix A. Supplementary data}

Supplementary data associated with this article can be found, in the online version, at http://dx.doi.org/10.1016/j.diabres. 2017.02.012.

\section{R E F E R E N C E S}

[1] Tremblay J, Hamet P. Biomarkers of vascular complications in type 2 diabetes. Metabolism 2015;64:S28-32.

[2] Groop PH, Thomas MC, Moran JL, Wadén J, Thorn LM, Mäkinen VP, et al. The presence and severity of chronic kidney disease predicts all-cause mortality in type 1 diabetes. Diabetes 2009;58:1651-8.

[3] Berk BC, Weintraub WS, Alexander RW. Elevation of Creactive protein in "active" coronary artery disease. Am J Cardiol 1990;65:168-72.

[4] Liuzzo G, Buffon A, Biasucci LM, Gallimore JR, Caligiuri G, Vitelli A, et al. Enhanced inflammatory response to coronary angioplasty in patients with severe unstable angina. Circulation 1998;98:2370-6.

[5] Emerging Risk Factors Collaboration, Kaptoge S, Di Angelantonio E, Lowe G, Pepys MB, Thompson SG, et al. Creactive protein concentration and risk of coronary heart 
disease, stroke, and mortality: an individual participant meta-analysis. Lancet 2010;375:132-40.

[6] Libby P. Inflammation in atherosclerosis. Arterioscler Thromb Vasc Biol 2012;32:2045-51.

[7] Saraheimo M, Teppo AM, Forsblom C, Fagerudd J, Groop PH. Diabetic nephropathy is associated with low-grade inflammation in Type 1 diabetic patients. Diabetologia 2003;46:1402-7.

[8] Jarvandi S, Davidson NO, Jeffe DB, Schootman M. Influence of lifestyle factors on inflammation in men and women with type 2 diabetes: results from the National Health and Nutrition Examination Survey, 1999-2004. Ann Behav Med 2012;44:399-407.

[9] Ros E, Martínez-González MA, Estruch R, Salas-Salvadó J, Fitó M, Martínez JA, et al. Mediterranean diet and cardiovascular health: teachings of the PREDIMED study. Adv Nutr 2014;5:330S-6S.

[10] van Bussel BC, Henry RM, Ferreira I, van Greevenbroek MM, van der Kallen CJ, Twisk JW, et al. A healthy diet is associated with less endothelial dysfunction and less low-grade inflammation over a 7-year period in adults at risk of cardiovascular disease. J Nutr 2015;145:532-40.

[11] Jaacks LM, Crandell J, Liese AD, Lamichhane AP, Bell RA, Dabelea D, et al. No association of dietary fiber intake with inflammation or arterial stiffness in youth with type 1 diabetes. J Diabetes Complicat 2014;28:305-10.

[12] Bernaud FS, Beretta MV, do Nascimento C, Escobar F, Gross JL, Azevedo MJ, et al. Fiber intake and inflammation in type 1 diabetes. Diabetol Metab Syndr 2014;6:66.

[13] van Bussel BC, Soedamah-Muthu SS, Henry RM, Schalkwijk CG, Ferreira I, Chaturvedi N, et al. Unhealthy dietary patterns associated with inflammation and endothelial dysfunction in type 1 diabetes: the EURODIAB study. Nutr Metab Cardiovasc Dis 2013;23:758-64.

[14] Strychar I, Cohn JS, Renier G, Rivard M, Aris-Jilwan N, Beauregard $\mathrm{H}$, et al. Effects of a diet higher in carbohydrate/ lower in fat versus lower in carbohydrate/higher in monounsaturated fat on postmeal triglyceride concentrations and other cardiovascular risk factors in type 1 diabetes. Diabetes Care 2009;32:1597-9.

[15] Bassuk SS, Rifai N, Ridker PM. High-sensitivity C-reactive protein: clinical importance. Curr Probl Cardiol 2004;29:439-93.

[16] Ahola AJ, Mikkilä V, Mäkimattila S, Forsblom C, Freese R, Groop PH. Energy and nutrient intakes and adherence to dietary guidelines among Finnish adults with type 1 diabetes. Ann Med 2012;44:73-81.

[17] Finnish Diabetes Association. Nutrition recommendation for a diabetic. Available from: http://www.diabetes.fi/files/308/ Ruokavaliosuositus.pdf. [23.09.16].

[18] Ahola AJ, Mikkilä V, Saraheimo M, Wadén J, Mäkimattila S, Forsblom C, et al. Sense of coherence, food selection and leisure time physical activity in type 1 diabetes. Scand J Public Health 2012;40:621-8.

[19] Wadén J, Tikkanen H, Forsblom C, Fagerudd J, PetterssonFernholm K, Lakka T, et al. Leisure time physical activity is associated with poor glycemic control in type 1 diabetic women: the FinnDiane study. Diabetes Care 2005;28:777-82.
[20] Thorn LM, Forsblom C, Fagerudd J, Thomas MC, PetterssonFernholm K, Saraheimo M, et al. Metabolic syndrome in type 1 diabetes: association with diabetic nephropathy and glycemic control (the FinnDiane study). Diabetes Care 2005;28:2019-24.

[21] Lopez-Garcia E, Schulze MB, Fung TT, Meigs JB, Rifai N, Manson JE, et al. Major dietary patterns are related to plasma concentrations of markers of inflammation and endothelial dysfunction. Am J Clin Nutr 2004;80:1029-35.

[22] Helmersson J, Vessby B, Larsson A, Basu S. Association of type 2 diabetes with cyclooxygenase-mediated inflammation and oxidative stress in an elderly population. Circulation 2004;109:1729-34.

[23] Wood AD, Strachan AA, Thies F, Aucott LS, Reid DM, Hardcastle AC, et al. Patterns of dietary intake and serum carotenoid and tocopherol status are associated with biomarkers of chronic low-grade systemic inflammation and cardiovascular risk. Br J Nutr 2014;112:1341-52.

[24] Ciubotaru I, Lee YS, Wander RC. Dietary fish oil decreases Creactive protein, interleukin-6, and triacylglycerol to HDLcholesterol ratio in postmenopausal women on HRT. J Nutr Biochem 2003;14:513-21.

[25] Mozaffarian D. Trans fatty acids - effects on systemic inflammation and endothelial function. Atheroscler Suppl 2006;7:29-32.

[26] Zapolska-Downar D, Siennicka A, Kaczmarczyk M, Kolodziej B, Naruszewicz M. Butyrate inhibits cytokine-induced VCAM1 and ICAM-1 expression in cultured endothelial cells: the role of NF-kappaB and PPARalpha. J Nutr Biochem 2004;15:220-8.

[27] Llauradó G, Gallart L, Tirado R, Megia A, Simón I, Caixàs A, et al. Insulin resistance, low-grade inflammation and type 1 diabetes mellitus. Acta Diabetol 2012;49:33-9.

[28] Dietrich M, Jialal I. The effect of weight loss on a stable biomarker of inflammation. C-reactive protein. Nutr Rev 2005;63:22-8.

[29] Hammonds TL, Gathright EC, Goldstein CM, Penn MS, Hughes JW. Effects of exercise on c-reactive protein in healthy patients and in patients with heart disease: a metaanalysis. Heart Lung 2016;45:273-82.

[30] Geffken DF, Cushman M, Burke GL, Polak JF, Sakkinen PA, Tracy RP. Association between physical activity and markers of inflammation in a healthy elderly population. Am J Epidemiol 2001;153:242-50.

[31] Ciccone MM, Aquilino A, Cortese F, Scicchitano P, Sassara M, Mola E, et al. Feasibility and effectiveness of a disease and care management model in the primary health care system for patients with heart failure and diabetes (Project Leonardo). Vasc Health Risk Manag 2010;6:297-305.

[32] Esposito K, Nappo F, Marfella R, Giugliano G, Giugliano F, Ciotola M, et al. Inflammatory cytokine concentrations are acutely increased by hyperglycemia in humans: role of oxidative stress. Circulation 2002;106:2067-72.

[33] Deleidi M, Jäggle M, Rubino G. Immune aging, dysmetabolism, and inflammation in neurological diseases. Front Neurosci 2015;9:172.

[34] Winkler JT. The fundamental flaw in obesity research. Obes Rev 2005;6:199-202. 